\title{
Percepción de los estudiantes que ingresan a estudiar pedagogía de lo que es un buen ciudadano, las instituciones públicas y la efectividad de la acción política*
}

\author{
CARLOS MUÑOZ LABRAÑA* \\ MARÍA PAZ CASANOVA LAUDIEN ${ }^{* * * *}$ \\ CECILIA BUSTOS IBARRA*** \\ ALFONSO HENRÍQUEZ RAMÍREZ ${ }^{* * * * *}$ \\ XIMENA GAUCHÉ MARCHETTT ${ }^{* * * * * *}$ \\ AMAYA ALVEZ MARÍN********
}

Universidad de Concepción - Chile

Recibido el 22-12-2016; primera evaluación el 01-08-2017; segunda evaluación el 17-08-2017; aceptado el 16-11-2017

* La investigación ha sido posible gracias al aporte realizado por la Dirección de Investigación de la Universidad de Concepción, Proyecto VRID N. ${ }^{\circ}$ 212.161.007-1.0.

Profesor de la Facultad de Educación de la Universidad de Concepción. Posdoctorado en Formación Ciudadana (Universidad de Valladolid), doctor en Educación (Universidad de Concepción), magíster en Historia (Universidad de Concepción), licenciado en Educación (Universidad de Concepción). Contacto: carlosem@udec.cl

Docente y jefe de Carrera de Ingeniería Estadística en la Universidad de Concepción. Estadística (Pontificia Universidad Católica de Valparaíso), magíster en Estadística (Pontificia Universidad Católica de Valparaíso) y doctora en Estadística (Pontificia Universidad Católica de Chile). Contacto: macasanova@udec.cl

${ }^{* * * *}$ Académica del Dpto. de Trabajo Social de la Facultad de Ciencias Sociales de la Universidad de Concepción. Doctora en Sociología (Universidad Autónoma de Barcelona), magíster en Salud Pública y Gestión Sanitaria (EASP y U-Granada, España), magíster en Trabajo Social y Políticas Sociales (Universidad de Concepción), trabajadora social (Universidad de Concepción). Contacto: cecbustos@udec.cl

${ }_{* * * * *}$ Académico de la Facultad de Ciencias Jurídicas y Sociales de la Universidad de Concepción. Licenciado en Ciencias Jurídicas Sociales (Universidad de Concepción), magíster en Filosofía Moral (Universidad de Concepción) y doctor en Derecho (Universidad de Chile). Contacto: alfhenriquez@gmail.com

${ }^{* * * * * *}$ Profesora asociada de la Facultad de Ciencias Jurídicas y Sociales de la Universidad de Concepción. Licenciada en Ciencias Jurídicas y Sociales (Universidad de Concepción). Doctora en Derecho (Universidad Autónoma de Madrid). Máster en Diplomacia y Relaciones Internacionales (Universidad Complutense de Madrid). Diplomada en Educación en Derechos Humanos (Instituto Interamericano de Derechos Humanos). Contacto: xgauche@udec.cl

Académica de la Facultad de Ciencias Jurídicas y Sociales de la Universidad de Concepción. Doctora en Derecho (PhD in Law, U. de York, Canadá). Magíster en Derecho (LL.M, U. de Toronto, Canadá). Licenciada en Derecho (U. de Concepción). Investigadora del Centro de Recursos Hídricos para la Agricultura y la Mineria CRHIAM. Contacto: Amaya.alvez@gmail.com 


\section{RESUMEN}

El artículo informa los resultados de una investigación que tuvo como objetivo estudiar las concepciones, con las que ingresan el universo de estudiantes de pedagogía a estudiar en la Universidad de Concepción, sobre lo que es un buen ciudadano, las instituciones públicas, y la efectividad de la acción política.

La investigación, de carácter cuantitativa y exploratoria, utilizó, como instrumento para recoger la información, la encuesta de conceptualizaciones, actitudes y acciones utilizada en la prueba de la International Association for the Evaluation of Educational Achievement (IEA), aplicada en Chile en 1999 y 2009 en la educación general (Mineduc, 2004).

Los resultados demostraron que los estudiantes, al comenzar sus estudios de pedagogía, manifiestan un bajo interés y comprensión de la política y, a la vez, una muy baja confianza en las instituciones públicas, como el gobierno, el congreso, la policía, los tribunales de justicia y los partidos políticos. Se destacan por la valoración que hacen de sus derechos, del rol del Estado y de las oportunidades que ofrece la democracia.

Palabras clave: percepciones, buen ciudadano, instituciones públicas, acción política.

\section{Perception of students who enter to study pedagogy of what a good citizen is, public institutions and the effectiveness of political action}

\section{Abstract}

The goal of this report is to study University of Concepcion preservice teachers' conceptions about what it means to be a good citizen, public institutions, and efficiency of the political action. The study was conducted by using an exploratory and quantitative method. Data about conceptions, attitudes and actions was gathered by using a test developed by the International Association for the Evaluation of Educational Achievement (IEA). In 1999, the test was applied to Chilean students and in 2009; the test was applied to primary and middle school students (Mineduc, 2004). Findings show that pre-service teachers have a low interest as well as comprehension about politics. In addition, they show very low confidence in public institutions such as government, congress, police, justice system, and political parties. On the other hand, they show high value regarding civil rights, the rol of the government and opportunities provided by democracy.

Keywords: perceptions, good citizen, public institutions, political action. 


\section{Percepçáo de estudantes que entram para estudar pedagogia do que é um bom cidadão, instituiçóes públicas e a eficácia da ação política}

\section{Resumo}

$\mathrm{O}$ artigo informa os resultados de uma pesquisa que teve como objetivo estudar as concepçóes com as que ingressam o universo de estudantes de licenciatura a estudar na Universidad de Concepcióm, sobre o que é um bom cidadão, as instituições públicas, e a efetividade da ação política.

A pesquisa, de caráter quantitativo e exploratório, utilizou como instrumento para colher a informação o questionário de conceitualizações, atitudes e ações utilizado no exame da International Association for the Evaluation of Educational Achievement (IEA), aplicada no Chile em 1999 e em 2009 na educação em geral (Mineduc, 2004).

Os resultados demonstraram que os estudantes, ao começar seus estudos em licenciatura, manifestam um baixo interesse e compreensão da política e, ao mesmo tempo, uma confiança muito baixa nas instituiçóes públicas como o governo, o Congresso, a polícia, os tribunais de justiça e os partidos políticos. Destacando-se pela avaliação que fazem dos seus direitos, do papel do Estado e das oportunidades que oferece a democracia.

Palavras-chave: percepçóes, bom cidadão, instituiçóes públicas, ação política.

\section{INTRODUCCIÓN}

La bibliografía especializada reconoce que el profesorado no es el único responsable de la formación de los ciudadanos, pues, como señala Bolivar (2007), la familia, los medios de comunicación y la sociedad en su conjunto también desempeñan un importante rol educativo en estas materias. Sin embargo, no es menos cierto que al profesorado le corresponden importantes responsabilidades de formar a los estudiantes en la comprensión del importante rol que les corresponde en la sociedad (Martínez, 2003).

Desde el retorno a la democracia, en nuestro país se han realizado esfuerzos por incorporar importantes temáticas ciudadanas hasta ese momento ausentes en el currículum escolar, lo que, además, situó a las asignaturas de "Lenguaje y Comunicación», "Historia, Geografía y Ciencias Sociales» y "Orientación» como aquellas que, en forma privilegiada, debían ocuparse del tema. Se generó también, de esta forma, un mapa de progreso que visibilizaba la temática a lo largo de toda la educación general (Muñoz y Torres, 2014).

A pesar de las importantes modificaciones efectuadas y aún de una serie de ajustes efectuados a partir del ańo 2004, la temática ciudadana ha tenido 
dificultades para poder instalarse en el sistema escolar. Varios son los factores que explican este fenómeno: algunos vinculados a falencias existentes en los propios procesos formativos realizados por el profesorado, mientras que otros están relacionados con una serie de percepciones, experiencias e ideas previas que poseen los propios profesores y alumnos acerca del sentido y de los alcances que se persigue con esta formación.

A nivel terciario, la tarea aparece aún más compleja, dado que, al parecer, el imperativo de formación profesional, en el cual están involucradas estas instituciones, no deja espacio curricular para estas y aún otras materias de formación general, a excepción de algunos programas de la responsabilidad social profesional con la que cuentan algunas instituciones en donde, tangencialmente, se abordan algunas de las temáticas asociadas.

El artículo que presentamos pretende adentrarse en la temática, investigando las percepciones que poseen los estudiantes en primer año de pedagogía acerca de lo que es un buen ciudadano, las instituciones públicas y la efectividad de la acción política.

La finalidad del estudio es cumplir dos objetivos fundamentales. Primero, generar un diagnóstico de aquellas percepciones con las que llegan los estudiantes desde la educación general a estudiar pedagogía a la universidad. Segundo, establecer una línea de base que contribuya a realizar los ajustes que sean necesarios a los procesos de formación inicial, con el objetivo de tomar todas aquellas decisiones que contribuyan a superar diversos prejuicios que poseen quienes, una vez egresados, tendrán la responsabilidad de contribuir a la formación ciudadana de sus futuros alumnos en la educación general.

\section{Marco teórico}

La preocupación por las relaciones de la ciudadanía con el estado y la cosa pública son de antigua data y, aunque el propio concepto de ciudadanía ha concitado la atención de numerosos analistas, especialmente en las últimas décadas, ha sido gracias a las aportaciones realizadas, desde una perspectiva histórica, por Marshall que estas relaciones han adquirido, nuevamente, notoriedad, al enunciar y vincular la ciudadanía a la existencia de distintos tipos de derechos que garantizan un mínimo de bienestar social (Marshall y Bottomore, 1998).

Un primer grupo de derechos corresponde a los civiles y políticos. Los derechos civiles incluyen aquellos vinculados a la libertad individual, de residencia, de expresión, de pensamiento y de confesión religiosa, así como de propiedad e igualdad ante la ley. Los derechos políticos incluyen la 
participación en la toma de decisiones, tanto al amparo de la institucionalidad pública como fuera de ella.

Un segundo grupo de derechos corresponde a los derechos sociales, vinculados a la seguridad, la educación y a un mínimo de bienestar económico, pudiéndose, en la actualidad, incluirse otros vinculados a la solidaridad y a lo colectivo, como el derecho de los pueblos al desarrollo y el de la libre expresión de los grupos indígenas.

Al situar los derechos sociales al mismo nivel que los civiles y los políticos, Marshall considera que la realización plena del ideal de ciudadanía es posible solo si todos los grupos de derechos gozan de la misma legitimidad y sus beneficiarios, los ciudadanos, son «iguales en cuanto a los derechos y obligaciones que ello implica» (Marshall y Bottomore, 1998, p. 23).

En el ámbito educativo, y, por tanto, de la forma en que los ciudadanos logran empoderarse de aquellos derechos que les permiten vivir en forma plena en la sociedad, Kerr (1999) distingue dos enfoques. El enfoque minimalista, reconocido por la literatura como el más clásico, se identifica con la educación cívica tradicional y posee una concepción restringida, superficial, excluyente, absolutista y descontextualizada de la ciudadanía y de la relación de esta con las instituciones públicas y la efectividad de la acción política. Desde el punto de vista formal, pone énfasis en el traspaso de ciertos conocimientos, especialmente declarativos: factuales y conceptuales, que son predefinidos por alguna autoridad, recayendo en el ciudadano su aceptación formal (Kerr, 2002). El enfoque maximalista, a diferencia del anterior, posee una concepción más profunda, inclusiva, comprensiva, contextual y amplia de la relación existente entre los ciudadanos, las instituciones públicas y la efectividad de la acción política. Se le identifica con el concepto de formación ciudadana y, desde el punto de vista formal, busca superar el traspaso de conocimientos puramente declarativos y descontextualizados, promoviendo el desarrollo de ciertas habilidades y actitudes que se esmera en construir de manera interactiva, hermenéutica y reflexiva entre los ciudadanos (Kerr, 2002).

Lo anterior lleva a que, como plantea Lizcano (2012), la ciudadanía no sea solo un atributo que confiere un estatus jurídico-político, sino que también, una condición indispensable que deben poseer aquellas personas e instituciones que se comprometen con su mejoramiento. Tarea en la cual es indispensable la generación y existencia de espacios de reflexión, participación y deliberación efectivos, en donde las unas y las otras, pongan en valor aquellos conocimientos, habilidades y actitudes que les permitan a los ciudadanos desenvolverse con éxito en la sociedad, especialmente, en la actualidad en que nos vemos enfrentados a una multiplicidad de desafíos políticos, económicos, 
sociales, culturales, medioambientales, migratorios, etc. Todo ello exige que los ciudadanos desarrollen capacidades que los ayuden a constituirse en partícipes inteligentes de aquellos debates que interesan a toda la comunidad (Nussbaum, 2005, p. 27).

Sobre este particular, y, a partir de un análisis del caso chileno, Paredes (2011) asigna una gran responsabilidad en estas materias, al diseño políticoinstitucional implementado por los gobiernos de la Concertación. La razón es que, a su juicio, este diseño incidiría en el déficit democrático y el creciente distanciamiento existente entre el espacio político y el social, que se expresaría en una descomposición, fragmentación y desarticulación del tejido social y en una «desafección y perdida de relevancia del espacio político para la ciudadanía» (Paredes, 2011, p. 491). Entre la cual, sin duda, los estudiantes y especialmente los futuros profesores poseen un rol fundamental.

He aquí donde adquiere valor el espacio educativo y el rol que corresponde al profesorado en este proceso. Sobre el particular, la literatura reciente reconoce que las concepciones de los estudiantes respecto al Estado y las instituciones públicas, la democracia y la ciudadanía y finalmente de los derechos de las mujeres e inmigrantes depende, como de ningún otro factor, de lo que ocurre en la escuela y lo que hace o deja de hacer en el aula el profesorado (Cerda, Egaña, Magendzo, Santa Cruz y Varas, 2004), pues son ellos quienes, en última instancia, traducen el currículo declarado en currículo aplicado.

A pesar de la importancia del profesorado, la investigación en el área demuestra que el espacio y el tiempo curricular que ocupan los conocimientos que responden a la racionalidad instrumental, sobrepasan con creces a los conocimientos que apuntan a una racionalidad axiológica y comunicativa tendiente a formar sujetos de derechos (Magendzo, 2004).

También existen otras evidencias que establecen que el componente ciudadano de la reforma educativa en Chile es uno de los menos comprendidos por los docentes y uno de los menos trabajados en el aula (Egańa, 2003), lo que pone en tela de juicio la capacidad del sistema escolar para promover aquellos conocimientos, habilidades y disposiciones ciudadanas que requieren para participar en la sociedad. Esta situación lleva a plantear a otros autores (Cerda et al., 2004), que, si no se implementan iniciativas explícitas acerca de la temática en el sistema escolar, especialmente en torno al sentido de la formación ciudadana, a los contenidos y las concepciones que sustentan las prácticas de los docentes en el aula, se hace muy difícil avanzar.

La dificultad fue constatada también por la Comisión de Formación Ciudadana (2004), al plantear que uno de los principales desafíos en la formación ciudadana de los estudiantes era ir más allá de la simple ilustración de 
ciertos derechos, pues se requería un énfasis en aquellos atributos, virtudes y valores que hacen a una persona un buen ciudadano, en el entendido que este concepto, así como otros, atiende precisamente a las disposiciones del carácter y a las destrezas intelectuales y prácticas que constituyen un ciudadano reflexivo, respetuoso y participativo.

Las aprehensiones de la Comisión de Formación Ciudadana (2004) se reflejan claramente en los resultados obtenidos en otra investigación efectuada en establecimientos de enseńanza básica de la Octava Región de nuestro país (Muñoz y Victoriano, 2009), en donde se constata que el profesorado no es capaz de intencionar, de manera expresa, las temáticas relacionadas con la formación ciudadana en sus clases, no se sienten interpelados por ellas y consideran que no poseen las herramientas mínimas para realizar con éxito su tarea en el proceso de enseñanza-aprendizaje. Los resultados obtenidos son coherentes con otra investigación efectuada en el mismo contexto que tuvo como propósito investigar las percepciones de los estudiantes (Muńoz y Torres, 2014), que arrojó como resultado que el estudiantado no posee una adecuada concepción de lo que es la formación ciudadana ni de lo que se persigue con ella, como tampoco que la escuela sea un espacio que los ayude en esta formación. Ello no quiere decir que los jóvenes no estén al tanto de los problemas sociales, ni que les deje de preocupar el entorno en que viven o vivirán más adelante (Rivera y Lissi, 2004), como ha quedado demostrado en Chile en los últimos años, a propósito de los movimientos estudiantiles de 2006 y 2011 (Rojas, 2012; Mayol, Rosenkranz y Ortiz, 2013 y PNUD, 2014).

En otra investigación que tuvo como propósito indagar la forma en que los profesores de historia comprenden la formación ciudadana en su ejercicio profesional y cómo la trabajan en sus clases (Reyes, Campos, Osandón, y Muńoz, 2013), se concluyó que, a pesar de que existe una gran diversidad en el tipo de formación entregada y en la comprensión sobre lo que es formar ciudadanos, en sus clases emplean estrategias muy homogéneas. Destacándose que los mencionados docentes, al momento de planificar sus clases, se basan antes en su experiencia de ejercicio ciudadano que en la formación académica recibida en la universidad.

A nivel latinoamericano Agudelo-Ramírez, Murillo-Saá, EcheverryRestrepo y Patińo-López (2013), en un estudio realizado en Colombia, concluyen que, en los jóvenes, predominan miradas adultocéntricas de reproducción de esquemas preestablecidos de lo que es un ciudadano, eludiendo el reconocimiento de prácticas emergentes y la creación de otras formas de ejercicio político. 
Wester (2008), a propósito del caso argentino, señala que, en este escenario, resulta interesante constatar que las escuelas perpetúan, en gran medida, estructuras y comportamientos autoritarios y represivos.

\section{Metodología}

La investigación fue de carácter cuantitativa y exploratoria. El instrumento utilizado fue la encuesta de conceptualizaciones, actitudes y acciones utilizada en la prueba de la International Association for the Evaluation of Educational Achievement (IEA), aplicada en Chile en dos ocasiones en la educación general (Mineduc, 2004).

En esta investigación la encuesta fue aplicada al universo de estudiantes que ingresaron a estudiar algunas de las 14 carreras de pedagogía ofrecidas en la Universidad de Concepción en el año 2014. La razón de trabajar a nivel universitario es que, dada la importancia que adquiere la educación ciudadana en la actualidad, ningún nivel educativo está exento de llevarla a cabo (Torres, Alvarez y Del Roble, 2013).

Las variables de interés se relacionaron con las percepciones de los estudiantes en relación con lo que es un buen ciudadano, las instituciones públicas y la efectividad de la acción política. Y, al igual que en las aplicaciones efectuadas en la educación general, la investigación parte de la base que la educación ciudadana en general y las temáticas que aborda la investigación en particular han sido incorporadas por los futuros profesores en distintos escenarios educativos formales e informales, siendo estos conocimientos la base de su acción futura, no solo en la sociedad, sino que también en los procesos formativos futuros y en su labor profesional, de allí, la importancia de conocer las percepciones que poseen los estudiantes al inicio de su formación profesional.

La investigación utiliza el modelo octogonal de Amadeo, Torney-Purta, Lehmann, Husfeldt y Nikolova, en el que el estudiantado aparece al centro circundado por distintos "portadores», cada uno de los cuales aporta, desde sus particulares puntos de vista, a sus diferentes concepciones (Amadeo, TorneyPurta, Lehmann, Husfeldt y Nikolova, 2002)

El modelo fue consensuado por los países participantes en el Estudio Internacional de 1999 y se sustenta en la teoría de la psicología ecológica del desarrollo y en la teoría cognitiva contextual. En el primer caso, los portadores (agencias) impactan al individuo a través de las relaciones cara a cara, siendo ejemplos de ello la familia, la escuela, el grupo de amigos (dentro y fuera de la sala de clase), la comunidad formal e informal y los medios de comunicación y la televisión. En el octágono más externo, dentro del cual están estos procesos que 
relacionan a la sociedad y al individuo, se encuentran los procesos, las instituciones y los valores políticos, económicos y educacionales o comunicacionales; la estructura de oportunidades y el sistema de estratificación socioeconómica; valores e instituciones religiosas; valores relativos a participación social (individualismo/colectivismo); la posición internacional del país, y los héroes, símbolos y narrativas nacionales o comunitarias (Mineduc, 2004). Cada uno de ellos impacta en la forma en que estas ocho dimensiones se reflejan en el discurso público y, en cierta medida, toma en cuenta las acciones y reacciones de los otros portadores y las características propias que poseen los individuos

En el segundo, es decir, la teoría cognitiva, el conocimiento consistiría en la capacidad para participar en las prácticas de la comunidad, para utilizar sus herramientas y para participar en los discursos de la sociedad, es decir, para interactuar e intercomunicarse, afectando y siendo afectado por los otros.

Ambos enfoques sugieren que los jóvenes se mueven de la participación periférica a la central en varias comunidades superpuestas (escuela/barrio), de tal manera que la socialización no consiste en que los adultos enseñen, sino que la comunidad misma rodea y crea las situaciones o contextos para desarrollar los conocimientos y la identidad del joven (Mineduc, 2004). Lo anterior hace que el estudio no solo reconozca la importancia de la escuela en estas materias, sino que también distinga la influencia que pueden ejercer otros portadores, como los especificados.

\section{Resultados}

La muestra consta de 396 estudiantes que ingresaron a estudiar alguna de las 14 carreras de Pedagogía de la Universidad de Concepción el año 2014, quienes accedieron a participar del estudio, por lo cual, algunas carreras tienen más baja representación de lo pre establecido mediante la estratificación proporcional ${ }^{12}$.

Se realizó un análisis de confiabilidad del instrumento, que llevó a una modificación de los reactivos en algunas dimensiones (cuyas dimensiones originales se presentan en la Tabla 1). Esto con el fin de lograr un instrumento con estándares adecuados de validez de constructo. Las dimensiones evaluadas y sus valores Alfa de Cronbach también se presentan en la Tabla 1.

\footnotetext{
$1 \quad 2$ En la Universidad de Concepción se ofrecen las siguientes carreras de pedagogía: Pedagogía en Educación Básica, Pedagogía en Educación Diferencial, Educación Parvularia, Pedagogía en Artes Plásticas, Pedagogía en Ciencias Naturales y Biología, Pedagogía en Ciencias Naturales y Física, Pedagogía en Ciencias Naturales y Química, Pedagogía en Educación Física, Pedagogía en Educación Musical, Pedagogía en Español, Pedagogía en Filosofía, Pedagogía en Historia y Geografía, Pedagogía en Inglés, Pedagogía en Matemática y Computación
} 
Tabla 1. Análisis de confiabilidad del instrumento

Dimensiones originales

Dimensiones nuevas

Alfa

Cronbach

A: Democracia

0,74

B: Buenos ciudadanos

0,76

C: Estado

0,80

D: Confianza en las instituciones

0,87

E: Oportunidades

0,80

F: El sistema político (Alfa $=0,54) \mathrm{F} 1$ : Visión respecto de la política

0,72

F2: Comprensión o interés en política

0,71

G: Currículo escolar

0,84

H: Discusión política

0,80

I: Acción política $2($ Alfa = 0,58)

I1: Acción política legal

0,72

I2: Acción política ilegal

0,83

J: Efectividad de la acción

J1: Efectividad de la acción

política $($ Alfa $=0,57)$ política

0,69

K: Uso de la fuerza militar

0,79

L: Atención a los medios de comunicación

0,73

Fuente: Elaboración propia con datos de los encuestados.

Las opciones de respuesta están en escala Lickert de 4 puntos expresados en conceptos. Para todas las dimensiones, se invirtieron los puntajes de los reactivos expresados en términos negativos, con el fin de que el valor 4 represente el mejor puntaje, dependiendo de la pregunta, por ejemplo, mayor comprensión, mejor opinión o predisposición.

Las dimensiones F (el sistema político) e I (acción política) presentaban cada una dos conceptos que no se asociaban adecuadamente, pero que al separarlos se logra la confiabilidad deseada.

En particular, para la dimensión I, cuenta tanto con reactivos vinculados a actividades legales como ilegales. Al no invertir los reactivos vinculados a actividades de acción política ilegal, la dimensión se encuentra correlacionada; mientras que al expresarlos todos en términos positivos la confiabilidad se pierde. Esto da cuenta de que los estudiantes no diferencias acciones políticas legales e ilegales al momento de involucrarse en ellas. Esto hizo necesario expresar la dimensión como dos conceptos diferentes (I1, I2). 
Ocurre algo semejante con la dimensión J, relativa a efectividad de la acción política. Solo el último reactivo se vincula actividades ilegales, pero la asociación se logra al no invertirlo, de donde se verifica nuevamente que los estudiantes no son capaces de diferenciar el tipo de acción política. Este reactivo se incorporó en la dimensión I2.

Se obtuvo, de esta manera, un mejor instrumento (cuyas dimensiones nuevas se presentan en la Tabla 1), respecto del cual se debe recordar invertir las preguntas F4, F5 y la sección J completa.

Tal como se expresa en la Figura 1, los resultados indican que los conceptos en los cuales se obtienen resultados negativos y son menos logrados por los estudiantes están vinculados a la comprensión e interés en la política, lo que quiere decir que los futuros maestros no comprenden que es, ni cuáles son los objetivos que persigue, a lo que se debe agregar que tampoco demuestran algún interés en ella. Resultados semejantes se obtienen al momento de interpelarlos acerca de su confianza en las instituciones, pues los estudiantes manifiestan abiertamente que no tienen confianza en las instituciones como el gobierno, el congreso, la policía, los tribunales de justicia y los partidos políticos. Lo que no quiere decir que los jóvenes tengan desinterés en conocer los problemas de su comunidad, o bien, posean aprehensiones en participar en otro tipo de organizaciones, solo que para ellos es poco llamativo participar en instancias sometidas a controles burocráticos (Navarro, 2005, p. 205).

Los resultados obtenidos ponen en tela de juicio la formación ciudadana recibida en la enseñanza media, pero también genera un tremendo desafío formativo a la educación superior en general y a la formación inicial profesional docente en particular. Esto porque esta debería ser capaz de colaborar para que los futuros maestros posean una adecuada visión sobre los temas en comento, por el importante rol formativo que les corresponde a estos futuros profesionales. El desafío no es menor, si se tiene presente que las mallas actuales de formación profesional no contemplan asignaturas obligatorias ni electivas destinadas a este fin, pues la mayoría del tiempo curricular está destinado a trabajar temáticas vinculadas a la disciplina que enseñarán, así como a los aspectos didácticos y evaluativos implicados en ellas.

Los ítems en donde los futuros maestros obtienen los mejores resultados son aquellos agrupados bajo el título de oportunidades que ofrece la democracia. Especialmente en los que hace referencia a la igualdad de oportunidades que brinda la democracia y en aquellos en donde se deben pronunciar acerca de los derechos que deben poseer las personas en una sociedad. Los estudiantes valoran la democracia como aquél sistema de gobierno que permite a las personas desarrollarse y vivir en forma plena en una sociedad, permitiendo un estado de derecho que valoran y propician sin prejuicios de género, religión, nacionalidad, condición socioeconómica ni cultural. 


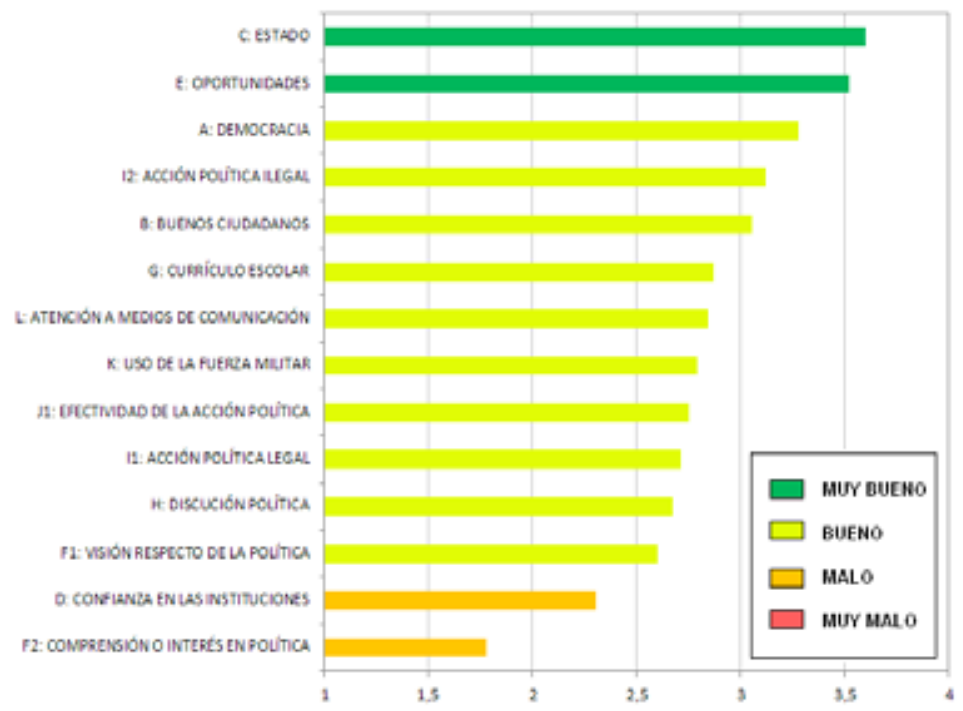

Figura 1. Evaluación global de actitudes y acciones cívicas de los estudiantes que ingresaron a estudiar Pedagogía en el año 2014 a la Universidad de Concepción. Fuente: Elaboración propia con datos de los encuestados.

Los futuros maestros, al momento de pronunciarse acerca del rol del Estado, logran, también, una valoración significativa, puesto que valoran la existencia de este ente permanente y garante de la institucionalidad, haciendo una clara distinción entre el rol que cumple el Estado con el de los gobiernos $\mathrm{y}$ las instituciones asociadas.

Desde otra perspectiva, al efectuar un análisis comparativo de los resultados obtenidos en las distintas dimensiones de la encuesta por carrera, no se encuentran diferencias significativas respecto a lo que es o no un buen ciudadano (dimensión B), del rol del Estado (dimensión C) ni tampoco al momento de consultar sobre la efectividad de la acción política (dimensión J1). Sin embargo destacan los resultados obtenidos por los futuros maestros de Pedagogía en Educación Musical con un puntaje significativamente mayor que el resto, al evaluar las respuestas vinculadas al concepto de democracia (A: Kruskall Wallis $\mathrm{p}=0,0078)$ y también los resultados obtenidos por los estudiantes de Pedagogía en Historia y Geografía, con un puntaje significativamente mayor en comprensión e interés en la política y comprensión de las repercusiones de las actividades vinculadas a la acción política ilegal (Kruskall Wallis $\mathrm{p}<0,0001$ ).

Este último hallazgo resulta interesante de destacar, debido a que los futuros maestros de Historia y Geografía poseen una responsabilidad 
preferente, aunque no exclusiva, en la formación ciudadana de los estudiantes en la educación general, existiendo un currículum que expresamente persigue estos propósitos. De tal manera que sus valoraciones son coherentes con lo que más tarde tendrán que enseñar.

En el polo opuesto, los resultados obtenidos por carrera dan cuenta de la obtención de puntajes significativamente inferiores a los demás de los futuros profesores de Pedagogía en Historia y Geografía, al momento de consultarles acerca de la confianza en las instituciones (Kruskall Wallis $\mathrm{p}<0,001)$. También los puntajes obtenidos por los estudiantes que ingresaron a estudiar Pedagogía en Ciencias Naturales y Biología, Inglés y Educación Física al momento de consultarles acerca de las oportunidades que brinda la democracia (Kruskall Wallis $\mathrm{p}=0,0361$ ) y respecto de la atención que brindaban a los medios de comunicación (Kruskall Wallis $\mathrm{p}=0,0240$ ). A los que se debe agregar los puntajes significativamente inferiores obtenidos por los estudiantes de Pedagogía en Ciencias Naturales y Biología y Educación Física en relación con la valoración que hacen de la participación en una discusión política (Kruskall Wallis p=0,0361); y por último la baja valoración que hacen los estudiantes de Pedagogía en Educación Física al momento de consultarles acerca de la posibilidad de participar en actividades de acción política legal (Kruskall Wallis p=0,0029).

La Figura 2 presenta los resultados obtenidos al comparar las actitudes y acciones cívicas por sexo.

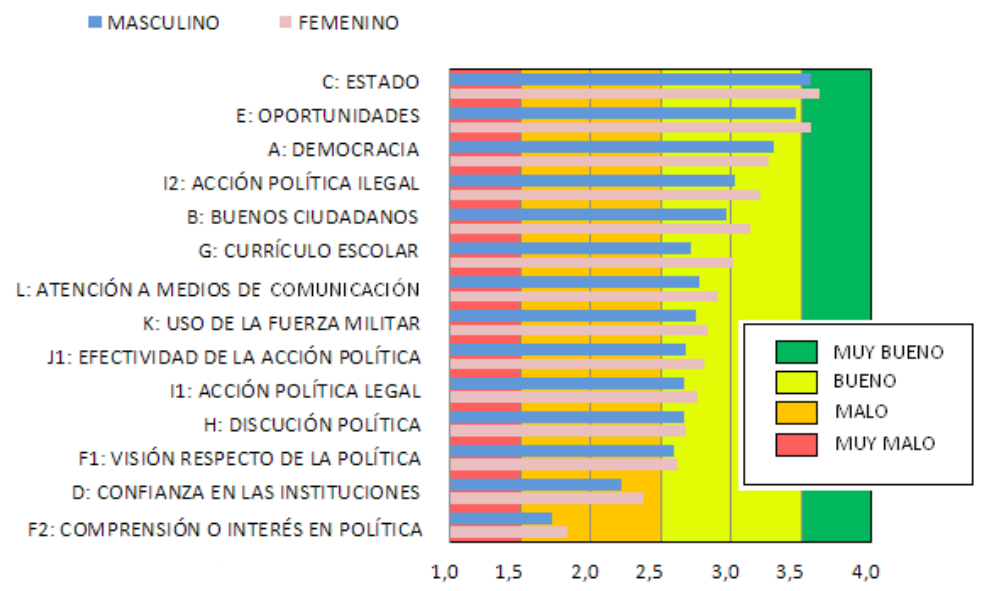

Figura 2. Comparación por sexo de Actitudes y Acciones Cívicas de los estudiantes que ingresaron a estudiar Pedagogía en el año 2014 a la Universidad de Concepción. Fuente: Elaboración propia con datos de los encuestados. 
Al comparar los resultados obtenidos por los estudiantes por género, se aprecia que en general existe significativamente mayor puntaje en las damas (Wilcoxon $\mathrm{p}<0,05$ ), excepto en el caso de las dimensiones relativas a la democracia, a su visión respecto de la política, a su comprensión o interés en la política y en la efectividad de la acción política, para las cuales no existe diferencia significativa entre los puntajes promedios obtenidos por ambos sexos (Wilcoxon $\mathrm{p}>0,05$ ).

En relación con los resultados obtenidos por edad, los opuntajes obtenidos por los estudiantes indican que para la mayoría de las dimensiones esta no influye sobre percepción que poseen los estudiantes en las dimensiones en estudio, con las siguientes excepciones. En relación con:

- La confianza en las instituciones, los resultados indican que esta decrece con la edad (Spearman; $\mathrm{p}=0,0044 ; \mathrm{r}=-0,15$ ).

- La posibilidad de participar en una discusión política, los estudiantes de mayor edad son más proclives a participar en ellas (Spearman; $\mathrm{p}=0,0100 ; \mathrm{r}=0,13$ ).

- La comprensión e interés en la política, los estudiantes de mayor edad puntúan más alto (Spearman; $\mathrm{p}=0,0070 ; \mathrm{r}=0,14$ ).

- A una posible participación en una acción política ilegal, los resultados indican que los estudiantes de mayor edad poseen mayor comprensión y conciencia de las repercusiones que pueden tener al involucrarse en ellas (Spearman; $\mathrm{p}=0,0480 ; \mathrm{r}=0,11$ ).

Por último, al comparar los resultados obtenidos, según la dependencia escolar del establecimiento de egreso de los estudiantes de la enseńanza media (particular, subvencionado o municipal), los resultados indican que no existen diferencias significativas entre unos y otros. Este hecho se repite al momento de analizar los resultados obtenidos por modalidad (científico humanista o técnico profesional). Dicho último hallazgo es relevante por cuanto contribuye a desechar el mito de la calidad de la formación recibida según la dependencia escolar de los establecimientos de egreso como de su modalidad.

\section{Conclusiones}

Los resultados obtenidos permiten realizar un diagnóstico de las actitudes y acciones cívicas con las que llegan los estudiantes que ingresan a estudiar las distintas carreras de pedagogía que ofrece la Universidad de Concepción. Pero, al mismo tiempo, constituyen una valiosa herramienta que permite establecer una línea base, que en calidad de desafíos se dejan entrever a los procesos formativos docentes que se podrían entregar o complementar en la universidad. 
De los resultados obtenidos, preocupa que los estudiantes no comprendan ni tengan interés por la política, no sean capaces de diferenciar entre una acción política legal e ilegal, (a excepción de los estudiantes de Pedagogía en Historia), y no tengan confianza en las instituciones (en donde los estudiantes de Pedagogía en Historia obtienen puntajes significativamente inferiores). Esto se debe a que la política y las instituciones asociadas conforman una base que garantiza el bien común, el «bien-estar» y la vida en comunidad y a la vez un eje a partir del cual, se puede dar solución a los problemas y desafíos que enfrenta la sociedad.

Mención aparte merece la valoración que hacen los estudiantes encuestados de las oportunidades que ofrece la democracia (en donde se destacan los estudiantes de Pedagogía en Educación Musical y preocupan aquellos que estudian Ciencias Naturales y Biología, Inglés y Educación Física), como medio para brindar oportunidades y garantizar ciertos derechos a las personas, así como del importante rol que asignan al Estado, como ente organizador de la sociedad. Esta idea supone que, para los estudiantes que participaron del estudio, existe un vínculo entre democracia y educación, en el sentido de entender a la democracia como una institución dinámica que requiere de la formación de ciudadanos activos que se comprometan con ella (Riba, 2006, p. 61). Una ciudadanía activa que es capaz de expandir la noción de democracia, desde aquel mínimo, pero esencial acto democrático que es el voto, hacia una relación más profunda del ciudadano con la acción política, generando una verdadera praxis democrática.

$\mathrm{Al}$ vincular la desafección con la política y la falta de confianza en las instituciones con la valoración que el mundo estudiantil efectúa de la democracia y del Estado, se produce un fenómeno muy particular. Puesto que, si bien los estudiantes manifiestan no tener interés en la política, valoran una forma específica de hacer política, aquella que brinda oportunidades y garantiza ciertos derechos a la población: la democracia. Una democracia que no vinculan a las instituciones como el gobierno, el congreso, la policía, los tribunales de justicia y los partidos políticos, sino que a un ente más permanente y superior como es el Estado.

Al valorar el Estado y la democracia, los estudiantes abogan por la existencia de políticas y compromisos más permanentes con la ciudadanía, que vayan más allá de los gobiernos, aunque sabemos que estos últimos son los responsables, en última instancia, de la buena ejecución y desarrollo de las políticas de Estado.

La disquisición es coherente con un fenómeno que ha venido ocurriendo en Chile en los últimos años, en donde se ha hecho común que en las reclamaciones ciudadanas se exija «más y mejor Estado», en detrimento de las acciones 
que pueden hacer, en la mejora de sus problemas, los gobiernos, los tribunales, la policía y la clase política.

También resultan interesantes los resultados obtenidos por género, ya que las mujeres obtienen puntajes superiores a los varones en todas las dimensiones, a excepción de aquellas vinculadas a la democracia y a la política (visión, interés y efectividad). El hallazgo no es menor y, de alguna manera, pone en evidencia la posibilidad que estas diferencias puedan explicarse por la mantención de los estereotipos socioculturales, que hacen que hombres y mujeres se sitúen y relacionen de manera distinta con la política, con la consiguiente falta de presencia de las mujeres en ella. Ello no deja de ser un desafío para nuestro país, pues, tal como da cuenta el Informe Auditoría a la Democracia, elaborado por el PNUD (2014), a pesar de que las mujeres representan más de la mitad del electorado nacional, la representación política de las mujeres en el poder legislativo alcanza al 13,9\%, porcentaje que nos ubica bajo el promedio mundial que es de $21,30 \%$ y bajo el promedio de la Región que asciende a $24,1 \%$. Esto no obstante que, en virtud de algunos compromisos internacionales, tales como la Convención sobre eliminación de toda forma de discriminación contra la mujer (CEDAW), Chile se ha comprometido a eliminar «todo concepto estereotipado de los papeles masculino y femenino en todos los niveles y en todas las formas de enseñanza» (artículo $10 \mathrm{c}$. CEDAW).

Por otro lado, si bien los resultados obtenidos por edad indican que esta no es una variable determinante en la valoración que hacen los estudiantes de lo que es un buen ciudadano, las instituciones públicas y la efectividad de la acción política, destaca el hecho de que, a mayor edad, los estudiantes posean una mayor comprensión e interés por la política, estén más dispuestos a participar en una acción en este ámbito y posean una mayor comprensión y conciencia de las repercusiones que puede tener su participación en una acción política ilegal. No obstante, a mayor edad, la confianza en las instituciones decrece. Lo anterior, junto con abrir una luz de esperanza, abre algunas interrogantes: ¿¿Será posible que la apatía de estos futuros profesionales con la política, sea solo una cuestión de edad?; ¿Qué rol deben cumplir los distintos niveles del sistema educacional?

\section{REFERENCIAS BIBLIOGRÁFICAS}

Agudelo-Ramírez, A., Murillo-Saá, L., Echeverry-Restrepo, L. y Patińo-López, J. A. (2013). Participación ciudadana y prácticas políticas de jóvenes en la cotidianidad. Revista Latinoamericana de Ciencias Sociales, Niñezy Juventud, 11(2), 587-602. https://doi.org/10.11600/1692715x.1129250612 
Amadeo, J., Torney-Purta, J., Lehmann, R., Husfeldt, V. y Nikolova, R. (2002). Civic knowledge and Engagement. An IEA study of upper secondary students in sixteen countries. Ámsterdam: International Association for the Evaluation of Educational Achievement. En Estudio Internacional de Educación Cívica. Santiago: Mineduc, 2004.

Bolívar, A. (2007). Educación para la ciudadanía. Algo más que una asignatura. Barcelona: Graó.

Cerda, A. M., Egaña, M. L., Magendzo, A., Santa Cruz, E. y Varas, R. (2004). El complejo camino de la formación ciudadana. Una mirada a las prácticas docentes. Santiago: LOM.

Egańa, L. (2003). Reforma Educativa y Objetivos Fundamentales Transversales. Los dilemas de la innovación. Santiago de Chile: PIIE.

Kerr, D. (1999). Citizenship education: An international comparison. National Foundation for Educational Research in England and Wales NFER/ qualifications and curriculum authority in England QCA. Londres: N.F.R.

Kerr, D. (2002). An International Review of Citizenship in the Curriculum: The IEA National Case Studies and the INCA Archive [Una Revista Internacional de Ciudadanía en el Plan de Estudios: Los estudios de caso de la AIE Nacionales y el Archivo INCA]. En G. Steiner-Khamsi, J. V. Torney-Purta y J. Schwille (eds.), New Paradigms and Recurring Paradoxes in Education for Citizenship: An International Comparison [Nuevos paradigmas y paradojas recurrentes en educación para la ciudadanía: una comparación internacional] (pp. 207-237). Ámsterdam: Elsevier Pres.

Lizcano, F. (2012). Conceptos de ciudadano, ciudadanía y civismo. Polis (Santiago), 11(32), 269-304. Recuperado el 01 de diciembre de 2014, de https://doi.org/10.4067/S0718-65682012000200014

Magendzo, A. (2004). Educación y ciudadanía: construyendo ciudadanía desde el currículum oficial y la cotidianidad de la escuela. Una lectura desde la investigación. PIIE Seminario Internacional. Reformas Curriculares en los noventa y la construcción de ciudadanía.

Marshall, T.H. y Bottomore, T. (1998). Ciudadania y clase social. Madrid: Alianza Editorial.

Martínez, J. (2003). Ciudadania, poder y educación. Barcelona: Graó.

Navarro, J. (2005) Las encuestas de jóvenes en Iberoamérica. Un recuento de experiencias recientes. Jóvenes, Revista de Estudios de la Juventud, 23, julio diciembre, 148-209.

Mayol, A., Rosenkranz, C. \& Ortiz, C. (2013). El Chile profundo: modelos culturales de la desigualdad y sus resistencias. Santiago: Liberalia Ediciones Ltda. 
Mineduc (2004). Educación Cívica y el ejercicio de la ciudadanía. Los estudiantes chilenos en el estudio internacional de Educación Civica. Santiago.

Muñoz, C. y Torres, B. (2014). La formación ciudadana en la escuela: problemas y desafíos. Revista Electrónica Educare, 18(2), 1-13. Recuperado de https:// doi.org/10.15359/ree.18-2.12

Muñoz, C. y Victoriano, R. (2009). El desafio de la Formación Ciudadana. Principios para un modelo de integración curricular en Lenguaje y Comunicación y Estudio y Comprensión de la Sociedad para la EGB. FONIDE F. 310894. Ediciones Universidad de Concepción.

Nussbaum, M. (2005). El cultivo de la humanidad: una defensa clásica de la reforma en la educación liberal (Juana Pailaya, trad.). Barcelona: Paidós.

Paredes, J. P. (2011). Ciudadanía, participación y democracia deuda y déficit en los 20 ańos de «Democracia» en Chile. Polis (Santiago), 10(28), 473-499. Recuperado el 01 de diciembre de 2014, de https://doi.org/10.4067/ S0718-65682011000100022

PNUD (2014). Informe Auditoría a la Democracia. Más y mejor democracia para un Chile inclusivo. Santiago: Programa de Naciones Unidas para el Desarrollo.

Reyes, L., Campos, J., Osandón L. \& Muñoz, C. (2013). El profesorado y su rol en la formación de los nuevos ciudadanos: desfases entre las comprensiones, las actuaciones y las expectativas. Estudios Pedagógicos (Valdivia), 39(1), 217-237. Recuperado el 01 de diciembre de 2014, de https://doi. org/10.4067/S0718-07052013000100013

Riba, J. (2006). Filosofía Política, República y educación del ciudadano. Res publica, 16(1), 61-76.

Rivera, N. y Lissi, M. (2004). La Responsabilidad Social: Cómo la Viven Tres Grupos de Estudiantes de Enseñanza Media en Chile. Psykhe [online], 13(2), 117-130 [citado 2015-05-12]. Disponible en: https://doi. org/10.4067/S0718-22282004000200009

Rojas, J. (2012). Sociedad bloqueada. Movimiento estudiantil, desigualdad y despertar de la sociedad chilena. Santiago: Ril Editores

Torres, A., Álvarez, N. y del Roble, M. (2013). La educación para una ciudadanía democrática en las instituciones educativas: Su abordaje sociopedagógico. Revista Electrónica Educare, 17(3), 151-172. Recuperado de http://www. revistas.una.ac.cr/index.php/EDUCARE/issue/current

Wester, J. H. (2008) Dimensiones y retos de una educación para la responsabilidad ciudadana. Utopia y Praxis Latinoamericana [online], 13(42), 55-69. [citado 2015-05-12], Disponible en http://www.scielo.org.ve/scielo.php?p id $=S 131552162008000300004 \&$ script=sci_arttext 